\title{
Der Aufstieg von Frankfurt/Rhein-Main zur europäischen Metropolregion
}

\author{
Eike W. Schamp, Frankfurt am Main
}

\section{Einleitung}

Weltstädte und global cities gelten in der modernen Globalisierungsdebatte als die Motoren, Kontroll- und Steuerungszentren von Vernetzungsprozessen, die kontinentale und globale Dimensionen besitzen. Eine differenzierte, nicht immer widerspruchsfreie hierarchische Ordnung scheint in diesem System von Städten zu bestehen (z.B. Friedmann 1995). Dieser Beitrag diskutiert die Rolle einer Metropolregion «zweiten» Grades. Der Begriff bezeichnet oft solche Stadtregionen, die bestenfalls Bedeutung für eine internationale Vernetzung im kontinentalen Maßstab haben. In dem jungen Prozess der verstärkten wirtschaftlichen Integration in Europa - gekennzeichnet durch den «Start» des Europäischen Binnenmarktes im Jahr 1993 und der Europäischen Währungsunion im Jahr 1994 stellt sich die Frage, welche der bestehenden nationalen Großstadt-Regionen zu Steuerungszentren von europäischer oder transkontinentaler Bedeutung aufsteigt. In dieser funktionalen Sichtweise müssen (metropolitane) Städte und ihre Region nicht als Territorien, sondern als Verortungen von Knoten, die über Entscheidungs-, Kontroll- und Koordinationsmacht verfügen, in weitreichenden Strömen verstanden werden. Castells hat sie als hubs in einem space of flows bezeichnet (CASTELLS 1996: 413). Städte und ihre Region gewinnen damit ihre Bedeutung im Stadtsystem durch die Reichweite und Vielfalt dieser Steuerungsfunktionen.

Frankfurt, oder genauer der Region Frankfurt/RheinMain, wird vielfach bereits der Status einer global city wegen seiner Bedeutung als Finanzplatz zugemessen (u.a. SAssen 1999, Keil \& Ronneberger 2000). Dies ist jedoch keineswegs ausgemacht. Zum einen ist die Stadt Frankfurt am Main mit etwa 0,65 Mio. Einwohnern alleine zu klein, um eine größere Vielfalt von Funktionen von zugleich bedeutendem Gewicht zu beherbergen. Erst die gesamte urbane Region verdient es, als Metropolregion bezeichnet zu werden. Zum anderen ist die Bedeutung einiger Funktionsbereiche möglicherweise begrenzt und die Vergrößerung ihrer Reichweite erst jüngsten Datums. Dieser Beitrag geht davon aus, dass Frankfurt/Rhein-Main sich erst in den letzten beiden Jahrzehnten schrittweise zum Standort verschiedener Knotenfunktionen von nationaler und internationaler Bedeutung entwickelt hat. Dabei werden zwei Thesen vertreten: Erstens entwikkelt sich im Prozess der europäischen Integration eine neue Spezialisierung einzelner Metropolregionen, die möglicherweise zu einer neuen Form der Hierarchisierung des nunmehr europäischen Stadtsystems beitragen wird. Doch folgt zweitens jede Spezialisierung und die darauf fußende Bildung der Metropolregion einem spezifischen Entwicklungspfad. Er kann als ein «emergenter» Prozeß verstanden werden, in dem sich Dimensionen des Nicht-Lokalen, nämlich Dimensionen des allgemeinen wirtschaftlichen Strukturwandels, der Veränderung politischer Bedingungen auf der nationalen und europäischen Ebene und des Aufkommens von «konkurrierenden» Metropolregionen, mit Dimensionen des Lokalen verbinden: Es besteht eine Vielfalt korrespondierender, kollaborierender und widerstreitender Handlungen mächtiger Akteure in der Metropolregion. Eine Folge ist die Konkurrenz verschiedener Funktionsbereiche in der Metropolregion um knappe regionale Ressourcen wie z.B. Fläche, «Humankapital» sowie öffentliche und politische Unterstützung, die die Vielfalt möglicher Knotenfunktionen begrenzt.

Eine Metropolregion wird damit zu einem «Spielfeld» ökonomischer Akteure in recht unterschiedlichen Themenfeldern - eben denen, die die ökonomische Spezialisierung der Region ausmachen. Der folgende Beitrag wird zunächst die Arena darstellen, in der sich verschiedene Spezialisierungen der Region entwikkeln. Er wird sich dann der Knotenfunktion in drei «Vernetzungssystemen» zuwenden: der Funktion als Knoten in der Generierung von Innovationen, in der Steuerung von Kapitalflüssen sowie in der Steuerung von Marktinformationen. Diese Knotenfunktionen werden sicher nicht durch Branchengliederung richtig erfasst; sie entsprechen vielmehr Wertschöpfungssystemen und erschließen sich daher stärker durch Cluster-Konzepte (etwa nach PORTER 1991). Die drei Knotenfunktionen der Metropolregion Frankfurt/Rhein-Main bestimmen durch ihre unterschiedliche Entwicklung in den vergangenen beiden Jahrzehnten den Entwicklungspfad von Frankfurt/ Rhein-Main, das den Weg von einem Zentrum nationaler Bedeutung zu dem einer europäischen Bedeutung nimmt.

Der Beitrag beruht auf Ergebnissen aus einem Forschungsprojekt über die regionale Entwicklung der Industrieforschung in Deutschland (finanziert durch die DFG; vgl. Specht 1998, 1999, Schamp 2001), aus der Mitarbeit am Sonderforschungsbereich 403 «Vernetzung als Wettbewerbsfaktor am Beispiel der Region Rhein-Main» (finanziert durch die DFG; vgl. Esser \& Schamp 2001) sowie aus dem Gemeinschaftsprojekt über «Emerging nodes in the global system: Tel Aviv and Frankfurt metropolitan areas compared" (mit A. Shachar von der Hebräischen Universität Jerusalem; 
finanziert durch GIF; vgl. Felsenstein, Schamp \& SHACHAR 2002).

\section{Die Arena: Die Entwicklung der Region zur internationalen Dienstleistungsmetropole}

Die Fülle der Berichte, die in den letzten Jahren von Sozialwissenschaftlern über die Region vorgelegt wurden, weist auf die hohe Entwicklungsdynamik von Frankfurt/Rhein-Main zum internationalen, wenn nicht globalen Zentrum hin (z.B. RONNEBERGER 1995, RONNEBerger \& Keil 1995, Sassen 1999, Felsenstein, Schamp \& Shachar 2002). Die Region Frankfurt/Rhein-Main - in der Abgrenzung, die sich in den 1990er Jahren in Wirtschaft und Öffentlichkeit durchgesetzt hat, und in der Bezeichnung des Wirtschaftsfördervereins umfaßt 15 Kreise sowie 7 kreisfreie Städte in drei Bundesländern und erstreckt sich von Limburg im W bis Aschaffenburg im $\mathrm{O}$, von der Wetterau im $\mathrm{N}$ bis zur Bergstraße im S. Es ist ein polyzentrischer Verdichtungsraum von etwa 4,8 Mio. Einwohnern - Tendenz langsam steigend - bei nur gering ansteigender, stark von Konjunkturen beeinflußter Erwerbstätigkeit. Mit 277 Mrd. DM Bruttowertschöpfung, d.h. etwa 8,5\% derjenigen Deutschlands, ist die Region eine wichtige und wohlhabende Produktionsregion im Lande (UVF 2000). Ihre Besonderheit liegt aber in dem außergewöhnlich hohen Grad der Internationalisierung. Indikatoren dafür sind die hohe und steigende Zahl von Ausländern (1999 13,9 \%, in der Stadt Frankfurt 24,1\%; vgl. auch FriedmanN \& Lehrer 1997) oder die große Bedeutung von hereinkommenden und herausgehenden Direktinvestitionen.

In der Region Frankfurt/Rhein-Main verbinden sich damit allgemeingültige Prozesse des Umbaus von Stadtökonomien in der Gegenwart mit denen einer wachsenden Internationalisierung. Dies soll kurz in drei Bereichen belegt werden: erstens der im Vergleich zu vielen anderen Großstadt-Regionen Deutschlands beschleunigten Deindustrialisierung; zweitens der schrittweisen Entwicklung von einem nationalen zu einem internationalen Dienstleistungszentrum; und drittens - damit verbunden - das hohe Maß an internationaler Verknüpfung sowohl der industriellen als auch der Dienstleistungs-Aktivitäten in der Region.

Dabei muss betont werden, dass der Entwicklungsprozess von Frankfurt/Rhein-Main zur Metropolregion von europäischer Bedeutung recht jung ist und sich erst in den 1980er und verstärkt 1990er Jahren entfaltet. Er greift dabei immer weiter in die Randbereiche der Metropolregion aus, sie auf diese Weise stetig vergrößernd. KeIL \& RONNEBERGER (1994) sahen gerade dort, an der Peripherie der Region, die internationalen Wachstumsspitzen. Ein Grund, nicht die Stadt Frankfurt allein als einen urbanen Knoten von europäischer Bedeutung zu sehen, sondern nur die gesamte Region.

\subsection{Deindustrialisierung}

Frankfurt und die Region gehörten zu den wichtigen Industrieregionen Deutschlands seit der Wende vom 19. zum 20. Jahrhundert. An der Spitze stand die Chemische Industrie, gefolgt von der Elektrotechnik, dem Maschinenbau und später der Autoindustrie. Erinnert sei an die führende Rolle, die Opel als Fahrrad-Fabrikant und späterer größter Autoproduzent in Deutschland spielte - bevor das Werk 1929 von General Motors übernommen wurde. Auch in der Nachkriegszeit wurde diese Industriefunktion gestärkt, unter anderem durch den Zuzug von AEG aus Berlin. Eine seit den 1950er Jahren fortdauernde Industrieverlagerung aus den Zentren an deren Rand bewirkt zusätzlich zum allgemeinen Rückgang der Industriebeschäftigung einen offen erkennbaren Deindustrialisierungsprozess in den städtischen Zentren, an deren Spitze Frankfurt (vgl. FREUND 1995). In vielen verbleibenden Industriebetrieben findet eine versteckte Deindustrialisierung statt, indem Funktionen der Produktion an extra-regionale Werke ausgelagert und Funktionen der Entwicklung, des Vertriebs etc. in der Region gestärkt werden.

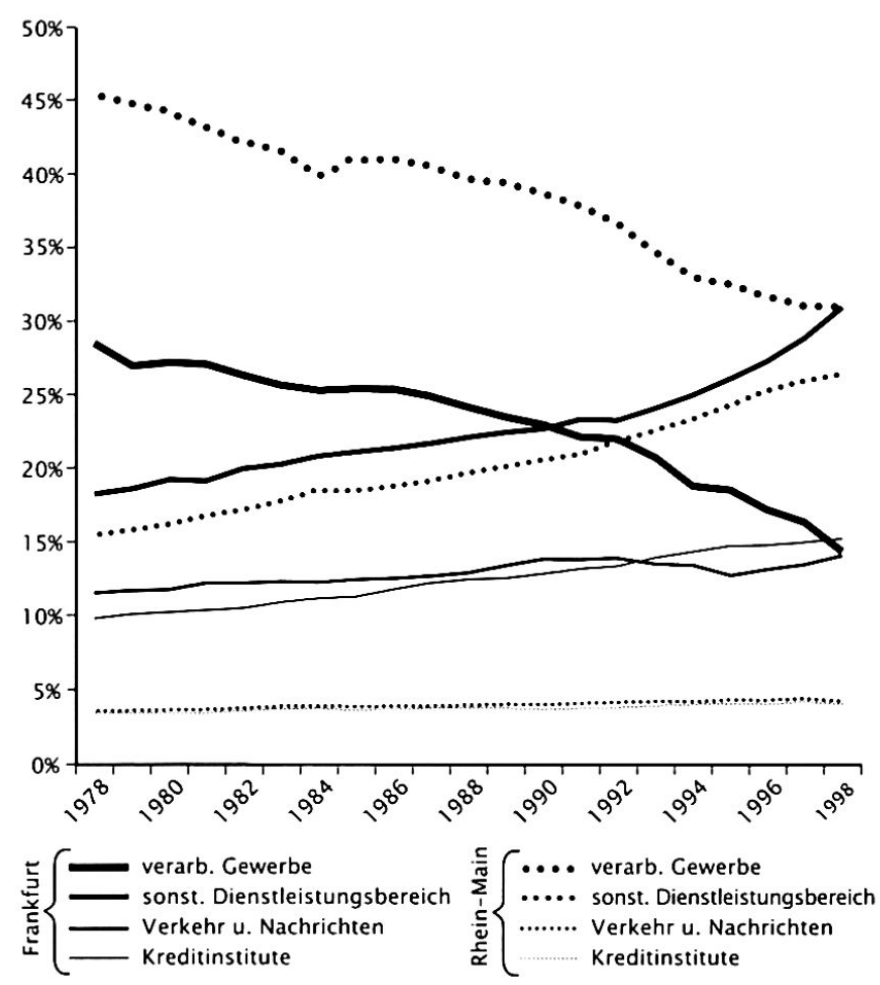

Abb. 1: Entwicklung der sozialversicherungspflichtig Beschäftigten nach Wirtschaftszweigen in Frankfurt und der Region Rhein-Main 1978 bis 1998

Sectoral employment in Frankfurt and Rhine-Main region 1978-1998

Evolution du nombre de détenteurs d'emploi assurés sociaux obligatoires, selon les branches économiques, à Francfort et dans la région du Rhin-Main (1978-1998) Quelle: Statistische Landesämter Bayern, Hessen und Rheinland-Pfalz 2000; Graphik: Ö. ALPASLAN 
In Frankfurt/Rhein-Main macht sich dies in einem erheblichen Rückgang der Industriebeschäftigung in den vergangenen beiden Jahrzehnten um $25 \%$ deutlich (Abb. 1). Bedenkt man, dass die genannten industriellen Cluster der Chemischen Industrie, der Elektrotechnik, des Maschinenbaus und der Autoindustrie dem 3. Kondratieff-Zyklus zugeordnet werden können und zugleich die Kernbranchen des fordistischen Zeitalters sind, dann müssen sich die Folgen des nach-fordistischen Umbaus gesellschaftlicher und wirtschaftlicher Strukturen in Deutschland in dieser Region konzentrieren. Diese Branchen gehören zwar zu den Stärken des Industrielandes Deutschland auf globalen Märkten, sind aber zugleich Branchen der mehr oder weniger ausgereiften Mitteltechnologien (NASCHOLD et al. 1998), die in den vergangenen Jahrzehnten teils erhebliche Krisen gesehen haben und einer starken Restrukturierung ausgesetzt sind.

\subsection{Tertiärisierung}

Die Stadt Frankfurt steht heute als Dienstleistungszentrale mit mehr als $83 \%$ der Beschäftigten (1998) in Dienstleistungsberufen an der Spitze in Deutschland. Anders als die Arbeiten über den Finanzplatz Frankfurt vermuten lassen, sind aber weniger die Arbeitsplätze im Kreditgewerbe gestiegen als vor allem diejenigen in den «sonstigen Dienstleistungsberufen» (Abb.1). Dahinter verbirgt sich eine Fülle verschiedenartiger Dienste, vor allem aber die für die Funktion eines nationalen und internationalen Steuerungszentrums wichtigen Bereiche der Rechts-, Unternehmens- und Finanzberatung, des Marketing und der Werbung, etc.. Während sich die Finanz-, Verkehrs- und non-profit-Dienstleistungen in den Städten der Metropolregion konzentrieren, ist dieser Bereich der sog. unternehmensbezogenen Dienstleistungen, der besonders zum Wachstum beiträgt, sowohl in den Städten als auch den Kreisen der Region verteilt. Der Handel dagegen hat sich vor allem in den nicht-urbanen Raum verlagert. Im Ganzen verlief die Tertiärisierung der
Metropolregion in den vergangenen beiden Jahrzehnten vor allem zugunsten der Städte und Gemeinden im Umland von Frankfurt (Tab.1).

In einigen Dienstleistung-Clustern geht das Wachstum langfristig mit einer Änderung der funktionalen Bedeutung der Region einher. Wie in den folgenden Abschnitten dargestellt, entwickeln sie sich zunächst zum $h u b$ für den nationalen Markt, was dann Voraussetzung für den Aufstieg zum hub im europäischen Maßstab ist. Zusammengenommen bestimmen sie damit den Entwicklungspfad der Metropolregion Frankfurt/Rhein-Main.

\subsection{Internationalisierung}

In der aktuellen Debatte um die Globalisierung von Städten und Regionen werden Direktinvestitionen als ein probates Messinstrument für die Darstellung der Bedeutung und Richtung von Steuerungsfunktionen betrachtet. Nun haben in der Region Frankfurt/RheinMain, lange bevor man über Globalisierung sprach, schon ausländische Unternehmen investiert. Zum Beispiel General Motors, die 1929 die Adam Opel AG in Rüsselsheim, damals den führenden deutschen Fahrrad- und Autohersteller, übernahmen (LOMPE et al. 1991). Andererseits hatten Unternehmen der Chemie ihrerseits im Ausland investiert - Investitionen, die in Folge der beiden Weltkriege wieder verloren gingen. Direktinvestitionen haben jedoch erst nach dem 2. Weltkrieg, in der langen Periode der pax americana, ihre heutige Bedeutung erhalten. Dies nicht allein wegen ihres enorm gestiegenden Umfangs, vor allem seit Mitte der 1980er Jahre (DiCKEN 1998), sondern auch wegen der völlig anderen, weil globalen Strategien, die heute hinter Auslandsengagements von Unternehmen stehen.

Richtung und Verhältnis von aus- und eingehenden Direktinvestitionen in eine Region vermögen die Rolle dieser Region als Knoten in internationalen Wertketten quantitativ zu beleuchten. Allerdings gibt es nur Daten für Bundesländer, nicht für einzelne Metropol-

\begin{tabular}{|llll|}
\hline Jahr & Stadt Frankfurt am Main übriges Rhein-Main-Gebiet & $\begin{array}{l}\text { Anteil der Stadt Frankfurt an allen } \\
\text { Dienstleistungsbeschäftigten der Region }\end{array}$ \\
\hline 1978 & 248,6 & 413,9 & 37,5 \\
1980 & 263,1 & 451,0 & 36,9 \\
1985 & 262,2 & 477,8 & 35,4 \\
1990 & 304,5 & 574,5 & 34,6 \\
1995 & 307,2 & 653,4 & 32,0 \\
1998 & 325,0 & 672,9 & 32,6 \\
\hline
\end{tabular}

Tab. 1: Die Entwicklung der Beschäftigung in allen Dienstleistungsbereichen in der Stadt Frankfurt und der übrigen Metropolregion von 1978 bis 1998 (in tausend)

Tertiary employment in Frankfurt and the rest of the metropolitan region 1978-1998

Evolution de l'emploi tertiaire dans la ville de Francfort et le restant de la région métopolitaine, en milliers (1978-1998)

Quelle: Statistische Landesämter Bayern, Hessen und Rheinland-Pfalz 2000 
regionen. Da Frankfurt/Rhein-Main etwa Dreiviertel der hessischen Wirtschaftskraft (gemessen in BWS) erwirtschaftet, können die Zahlen für Hessen dennoch näherungsweise die Bedeutung der Region beschreiben: Bezogen auf die Höhe der hereinkommenden Auslandsinvestitionen je Beschäftigten steht Hessen in Deutschland seit langem an der Spitze.

Die Kapitalbeziehungen der Metropolregion Frankfurt/Rhein-Main zum Ausland unterscheiden sich signifikant vom Durchschnitt Deutschlands: Während die herausgehenden Investitionen der deutschen Wirtschaft schon seit Beginn der 1980er Jahre die hereinkommenden übertrafen, wuchsen beide in Hessen bis zu Beginn der 1990er Jahre im Gleichklang. Entgegen den Klagen über die nachlassende Attraktivität des «Standortes Deutschland» blieb also die Region noch länger für ausländische Investoren attraktiv. Erst seit Beginn der 1990er Jahre übertrafen auch in Frankfurt/ Rhein-Main die herausgehenden Investitionen zunehmend die hereinkommenden. Noch interessanter ist

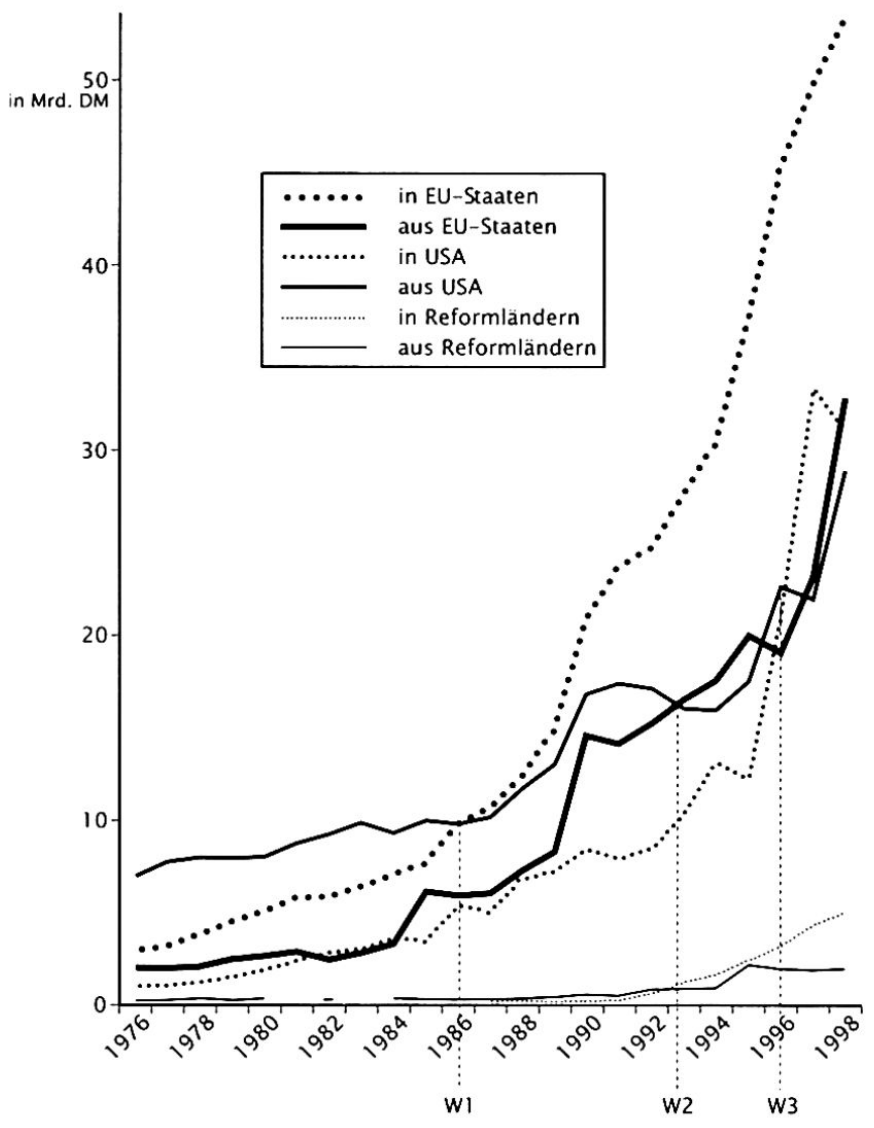

Abb. 2: Die Entwicklung der Direktinvestitionen nach und aus Hessen 1976 bis 1998 nach Herkunfts- und Zielländern

Foreign direct investment to and from the Land of Hesse according to countries 1976-1998

Evolution des investissements directs étrangers dans le Land de Hesse et des investissements directs hessois à l'étranger (1976-1998)

Quelle: Deutsche Bundesbank; Graphik: Ö. AlpasLan die Entwicklung der Beziehungen zu einzelnen Großregionen der Weltwirtschaft, die durch drei «Wendepunkte» markiert wird (Abb. 2): Mitte der 1980er Jahre begannen die herausgehenden Investitionen in EUStaaten die hereinkommenden, seit langem dominanten Investitionsströme aus den USA zu übertreffen. Anfang der 1990er Jahre übertrafen dann die aus EU-Staaten kommenden Direktinvestitionen diejenigen aus den USA. Mitte der 1990er Jahre schließlich übertrafen die in die USA gehenden Direktinvestitionen diejenigen aus den USA und aus den EU-Staaten. Diese Entwicklung belegt unsere These, dass sich die Region von einem nationalen Platz, auf dem unter den Ausländern amerikanische Unternehmen dominierten, zu einem Kontrollzentrum von europäischen Kapitalströmen entwickelte.

\section{Die «ungesicherte» Stellung der metropolitanen Steuerungsfunktionen}

Die Rolle eines urbanen Agglomerationsraumes als metropolitaner Raum wird im allgemeinen durch die Art und Reichweite der Steuerungsfunktionen bestimmt, die von dieser Region ausgehen und einen internationalen Raum erreichen. Frankfurt/Rhein-Main steht dabei in der Spannung zwischen «globalen» Funktionen, die im allgemeinen mit der Funktion des Finanzplatzes gleichgesetzt werden (z.B. SASSEN 1999), und «europäischen» Funktionen. Aus unserer Sicht überwiegen die letzteren bei weitem. Um den Entwicklungspfad einer Metropolregion bestimmen zu können, müssen Steuerungsfunktionen ein besonderes Gewicht haben, das sich auch in der Bedeutung für den regionalen Arbeitsmarkt und den regionalen Wohlstand artikuliert. Sie sind daher i.a. nur in wenigen "Clustern» der metropolitanen Ökonomie anzutreffen.

Frankfurt/Rhein-Main gilt weithin als einer der wohlhabendsten Räume Europas, als ein prosperierender urbaner Raum, der durch stetige Zuwanderung von Menschen und Unternehmen und sich modernisierende ökonomische Strukturen gekennzeichnet ist. Die folgenden Abschnitte sollen die These belegen, dass diese Eigenschaften keineswegs unumstritten sind, dass also die Rolle der Metropolregion als ein wichtiger Knoten im europäischen Wirtschaftsraum keinesfalls gesichert ist. Sie ist vielmehr in vielem prekär, und das einerseits, weil es zu Konflikten in der Region um die Stellung der Ressourcen kommt, die für die Bewahrung dieser Rolle als notwendig erachtet werden, und andererseits, weil andere metropolitane Räume in Europa im «Management» erfolgreicher scheinen und daher für mobile Unternehmen attraktiver werden können.

\subsection{Frankfurt/Rhein-Main als Innovationszentrum} Zufolge der aktuellen Innovationsforschung sind Metropolregionen zentrale hubs für die Generierung 
und die Diffusion von Wissen und Innovationen (vgl. z.B. Feldman \& Audretsch 1999). In der fordistischen Organisation von Großunternehmen war - und ist teilweise noch - das Zentrum der industriellen Forschung und Entwicklung direkt der Hauptverwaltung eines Unternehmens beigegeben und beide haben ihren Standort in einer Metropolregion. Im Zeitalter postfordistischer Organisation werden lokal vernetzte Innovationsinseln durch transnationale Unternehmen in deren globales System der FuE eingebunden (SPECHT 1999). Auch diese Inseln werden überwiegend in großen städtischen Regionen vermutet. Für die Region Frankfurt/Rhein-Main trifft eher die Form der fordistischen Organisation von FuE zu, deren gegenwärtiger Um- und Abbau die Rolle der Metropolregion als gesamte Innovationsinsel in Frage stellt (im einzelnen vgl. Schamp 2001). Die Region ist eine der elf europäischen «Innovationsinseln» (EU 1994), in der sich sowohl das FuE-Personal (SPECHT 1999) als auch die Patentanmeldungen (Greif \& SchmiedL 1998) konzentrieren. Dies ist das Erbe der Industrialisierung in besonders forschungsintensiven Industrie-Clustern der chemischen, elektrotechnischen und Autoproduktion. Ein Blick auf die Entwicklung der industriellen Forschung und Entwicklung (FuE) zeigt die Tendenzen, die die gesamte metropolitane Innovationsinsel gegenwärtig nimmt:

- Die Chemieforschung dominiert zwar weiterhin in der Region. Aber die Auflösung des Hoechst-Konzerns, die Fusion wichtiger Teile mit der französischen Rhône-Poulenc und die anschließende Abwanderung des fusionierten Konzerns Aventis nach Straßburg bzw. Lyon in den 1990er Jahren hat gravierend zum Abbau der Chemieforschung in der Region beigetragen. Der Anteil von $44 \%$ aller FuEBeschäftigten der Region in der Chemie im Jahr 1993 ist seitdem erheblich gefallen, da Hoechst allein davon mehr als $30 \%$ stellte. Noch gibt es allerdings andere forschungstreibende, «mittelständische» Chemieunternehmen in der Region - etwa Merck oder Wella in Darmstadt oder BoehringerIngelheim. Ausländische Chemie-Unternehmen haben wenig in die FuE in der Region investiert bzw. kürzlich sogar des-investiert. In einer Umfrage unter den 45 größten ausländischen Chemieunternehmen in der Region fand SPECHT (1998) nur sieben mit eigener FuE-Abteilung, die jedoch überwiegend für die Marktbeobachtung und -anpassung tätig waren. Symptomatisch ist das Beispiel Du Pont de Nemours: Das amerikanische Unternehmen hatte Anfang der 1990er Jahre mit 30 Mio. DM Investition ein spezielles FuE-Zentrum bei der Deutschlandzentrale (Bad Homburg) angelegt, jedoch wenige Jahre später wieder geschlossen.

- Die Autoindustrie und ihre Zulieferer sind in Deutschland zum führenden Cluster der Industrieforschung herangewachsen: Auf sie entfielen
$41 \%$ aller FuE-Aufwendungen der deutschen Industrien im Jahr 1999, mit weiterhin steigender Tendenz (Wissenschaftsstatistik 2000). Im neo-fordistischen Umbau des Automobilclusters erstreckt sich der Prozess des outsourcing zufolge der schlanken Produktionskonzepte nicht nur auf die Produktion, sondern auch die FuE. Wachsender Wettbewerbsdruck auf stagnierenden Märkten erzwingt höheren FuE-Aufwand, so dass auch die konzern-eigenen Entwicklungszentren mitwachsen. Jede Region, die Standort eines Auto-Unternehmens ist, das über eigene Entwicklungskompetenz verfügt, profitiert von dieser Entwicklung.

Folglich profitiert die Region Frankfurt/Rhein-Main von der Stärkung der FuE-Funktionen im Internationalen Entwicklungszentrum ITEZ der Opel AG, dem bis vor kurzem durch General Motors die Aufgabe zugewiesen war, alle GM-Modelle außerhalb der beiden Amerika zu entwickeln. Die Anzahl der im ITEZ in Rüsselsheim Beschäftigten stieg im vergangenen Jahrzehnt auf weit mehr als 8.000, ergänzt durch jeweils hunderte in benachbarten speziellen Forschungszentren von Opel sowie in Unternehmen der technischen Beratung («Ingenieurbüro»), deren führende Firmen sich in den 1990er Jahren dort angesiedelt haben (vgl. RENTMEISTER 2001). Weitere FuE-Beschäftigung ist bei vielen, insbesondere den großen amerikanischen Zulieferern entstanden. In Frankfurt/Rhein-Main befindet sich zudem das europäische Entwicklungszentrum der meisten japanischen Autohersteller: Honda, Mazda, Mitsubishi und Subaru sind hier vertreten. Auch wenn infolge der Vielfalt von Zulieferbranchen der gesamte Umfang dieser FuE-Kapazitäten nur schwer messbar ist, wird doch deutlich, dass die Region eine wichtige «Innovationsinsel» im Themenfeld Auto-Technik in Europa ist.

Die Industrieforschung in den weiteren Clustern der Elektrotechnik und des Maschinenbaus, die ehemals für die Region von großer Bedeutung waren, ist jedoch weitgehend mit ihren Unternehmen untergegangen. Für den Untergang stehen einstmals so bekannte Namen wie AEG oder auch Pittler (Maschinenbau), die in der Krise Mitte der 1990er verschwanden.

Die vorherrschenden Technologiefelder der industriellen Innovationsinsel Frankfurt/Rhein-Main sind damit weitgehend in sog. Mitteltechnologie-Branchen angesiedelt. Politiker in Land und Stadt erkannten dies anfangs der 1990er Jahre als Zukunftsschwäche. Eine von Stadt und Umlandverband in Auftrag gegebene Studie stellte die Bedeutung von Hochtechnologie für die regionale Entwicklung heraus und identifizierte verschiedene Technikfelder (UVF 1993), aus denen die Politiker von Land und Stadt vor allem die Biotechnologie als neue Hoffnung auswählten. In verschiedenen Initiativen beteiligte sich die Region unter Führung der Stadt und mit Hilfe des Landes Hessen 
am Bundeswettbewerb BioRegio (und verlor, vgl. u.a. DoHsE 2000), später am neuen Wettbewerb BioProfile (und gewann); das Land errichtete gemeinsam mit der Hoechst AG einen venture capital fonds, die GoetheUniversität schuf u.a. ein neues Institut für Bioinformatik, ein Max Planck-Institut wurde verlagert, und schließlich errichtet die Stadt einen Biotechnologiepark. Während sich hier zum ersten Mal ein lokales Netzwerk im Sinne eines lokalen Innovationssystems zu entwickeln scheint, mehren sich kritische Stimmen über die Schwierigkeiten des Unterfangens: Die chemische Industrie sei zu wenig beteiligt, es würden zu wenig innovative produzierende Unternehmen entstehen, und außerdem käme man zu spät im Vergleich zu München und anderen Biotechnologie-Regionen Deutschlands. Demgegenüber hat sich im südlichen Teil der Region um Darmstadt erfolgreich ein neuer Technologieschwerpunkt der Telekommunikation und Software entwickelt. Er ist jedoch bislang überwiegend nur von nationaler Bedeutung, verglichen mit München als «Germany's No.1» (STERnBerg/TAmÁsy 1999).

Die «europäische Innovationsinsel» Frankfurt/RheinMain scheint damit ihre bisherige Bedeutung schrittweise einzubüßen.Zudem ist die Region nicht mehr der Ausgangspunkt der Entscheidungsmacht, wie durch die Abwanderung von Hoechst belegt, sondern wird zunehmend zum Verankerungspunkt von Teilen fremder transnationaler Unternehmen, wie in der Chemie und besonders im Automobilsektor deutlich wird.

\subsection{Finanzplatz Frankfurt}

Die Bedeutung Frankfurts als Finanzplatz auf «globalen» Finanzmärkten ist inzwischen vielfach diskutiert worden (jüngst z.B. SASSEN 1999, Grote 2002, Lo \& Schamp 2001). Doch bleiben die Meinungen geteilt:

- Erstens gibt es weder eine allgemeine Theorie des Finanzplatzes noch allgemein akzeptierte Kriterien, wie ein Finanzplatz gemessen werden soll. So bleibt es bei der Zahl der Banken und vor allem Auslandsbanken, mit deren Hilfe die Bedeutung eines Platzes als Entscheidungs-Knoten in Finanzströmen (unzureichend) beleuchtet wird. Während sich die deutschen Banken in Frankfurt konzentrieren - ihre Zahl allerdings angesichts von Fusionen abnimmt -, liegt die Zahl der Auslandsbanken mit 337 zwar hinter der global (finance) city London (429), aber vor derjenigen von New York (237) (GROTE 2002). Seit Beginn der 1980er Jahre sind stetig knapp 70\% aller Auslandsbanken in Deutschland in Frankfurt lokalisiert. Auslandsbanken führen allerdings zumeist nicht selbst Geschäfte aus, sondern sind "Anbahnungs- und Kontaktstellen». Ein ebenso unzureichender Indikator ist die Zahl der Beschäftigten: Sie macht in Frankfurt ca. 68.000 und in der gesamten Metropolregion ca. 125.000 (1998) aus. Damit übersteigt sie zwar alle anderen (Finanz-) Städte in Deutschland (Schamp 1999). Aber einer- seits überschätzt sie die Bedeutung des Finanzplatzes, weil auch die Beschäftigten «am Bankschalter» darin enthalten sind, andererseits unterschätzt diese Zahl die Bedeutung, weil nicht alle Beschäftigten der im Finanzcluster verbundenen Branchen erfaßt werden (vgl. Lo \& Schamp 2001). Versteht man nämlich einen Finanzplatz als einen Ort, an dem Finanzprodukte «gemacht» werden, dann bedarf es dazu einer Vielfalt an informierenden, beratenden (von Reuters bis zu Anwälten und Unternehmensberatern) und technischen Dienstleistungen der Informationstechnologie.

- Zweitens unterliegt ein Finanzplatz gegenwärtig in besonderem Maße widerstreitenden Tendenzen der räumlichen Dezentralisierung und Konzentration. Die Finanzplatz-Literatur mißt der Größe einer Börse erhebliche Bedeutung zu. In der Tat unterliegt die Marktplatz-Funktion für standardisierte Finanzprodukte (z.B. Dax-Aktien) einer erheblichen Tendenz zu Skaleneffekten: je größer, umso mehr Liquidität, umso «bessere» Marktergebnisse. Folglich scheint ein erheblicher Konzentrationsprozess auf wenige (oder nur einen) Börsenplatz zu entstehen. Tatsächlich hat sich Frankfurt zunächst in Deutschland als Börsenplatz durchgesetzt (Schamp 1999) und dann Ende der 1990er Jahre beschleunigt eine europäische Bedeutung gewonnen. Die zur Erzielung ökonomischer Skalenerträge eingesetzte Technik des DV-gestützten remote access lässt damit den Organisator der Börse (Deutsche Börse AG) als Gewinner in Europa erscheinen, nicht jedoch den «Platz». Denn nun handeln vermehrt Londoner Händler von London aus auf der «Frankfurter» Börse (im einzelnen Grote 2002).

Dieser dezentralisierenden Tendenz stehen konzentrierende Tendenzen auf Teilmärkten gegenüber, die es mit wenig transparenten «Finanzprodukten» bzw. Erwartungen zu tun haben. Sowohl im Investmentbanking als auch der Vermögensverwaltung bedarf es vielfältiger Formen der Information und Beratung («Analyse», «Research», Rechts-Beratung) zur Herstellung eines Finanzproduktes, so dass Formen der Kommunikation zwischen Akteuren notwendig werden, die Aktualität, Ausschließlichkeit und Interpretation bereit stellen; folglich macht dies den persönlichen Kontakt in räumlicher Nähe erforderlich (SASSEN 1999, Lo 2001, Lo \& Schamp 2001).

Finanzplätze stehen in stetiger Konkurrenz untereinander - vor allem dann, wenn die Finanzmärkte u.a. durch Deregulierung und Reregulierung schnellen Veränderungen unterliegen. Die Rolle Frankfurts als ein wichtiger europäischer hub in Finanzströmen heute gilt es als der zweite europäische Finanzplatz nach London, noch vor Paris - ist das vorläufige Ergebnis eines langen Entwicklungspfades, in dem Frankfurt zunächst nach dem 2 . Weltkrieg langsam als führender deutscher Finanzplatz heranwuchs, um seit Beginn der 
1980er Jahre, beschleunigt in den 1990er Jahren eine zunehmend wichtige Rolle in Europa zu spielen (vgl. Grote 2002, Schamp 1999). Diese Rolle ist jedoch immer noch eng mit der Größe und Stärke der deutschen Volkswirtschaft verknüpft und daher nicht im eigentlichen Sinne «global»: Zwar wird zunehmend von Frankfurt aus durch die Europäische Zentralbank die nationale Finanzpolitik und Geldpolitik in Europa beeinflusst, aber für die privaten Finanzdienstleister ist Frankfurt im wesentlichen der Ort, den großen deutschen Markt zu erreichen (Grote 2002).

\subsection{Der logistische hub Frankfurt}

Ein wenig übersehen und noch wenig erforscht ist die veränderte Rolle der Region Frankfurt/Rhein-Main in Waren- und Informationsströmen, die die klassische Aufgabe von Städten - die Distribution - betreffen. Die «Globalisierung» als Ausdruck einer veränderten Reichweite von Warenströmen und zugleich als Ausdruck einer Verlängerung von Wertketten - so dass immer mehr «intermediäre» Produkte gehandelt werden - macht vielfach die Neudefinition der Rolle des gateway zu territorialen Märkten notwendig. Frankfurt/Rhein-Main entwickelt sich stärker als zuvor zu einem führenden gateway für zentral-europäische Märkte, neben den unmittelbaren Nachbarländern Niederlande, Österreich und Schweiz vor allem Osteuropa (vgl. Felsenstein, Schamp \& Shachar 2002). Einerseits wandert die Drehscheiben-Funktion in der eigentlichen (Transport-)Logistik langfristig von den Seehäfen zu Binnenstandorten. Frankfurt/Rhein-Main gilt als der Gewinner dieser Prozesse (LÄPPLE 2000), obwohl neuerdings die Rolle als hub in der terrestrischen physischen Warenverteilung angesichts hoher Bodenpreise und zunehmender Verkehrsprobleme zunehmend unter Druck gerät (BERTRAM 1994). Lagerhaltung und Waren-Transport werden mit tatkräftiger öffentlicher Unterstützung an neue Standorte im ländlichen Raum von dennoch zentraler Lage (Hersfeld, Bebra) verlegt. Andererseits nimmt die Bedeutung von Steuerungsfunktionen der Distribution, also der nicht-physischen Funktionen in der Warenverteilung, erheblich zu. Disposition in Speditionsunternehmen oder in den Deutschland-Zentralen ausländischer Unternehmen konzentrieren sich in Frankfurt/RheinMain und stärken dadurch die Büro-Funktionen der Region.

In diesen Marktplatz-Funktionen wird die Bereitstellung von Informationen über Marktentwicklungen und Anbahnungs-Möglichkeiten für die Gewinnung neuer Kunden zu einem bedeutenden Faktor der geographischen Konzentration in einer Metropolregion. Diese wird nicht allein durch die Anzahl von Vertriebs-Einrichtungen in der Metropolregion bewirkt. Messegesellschaften, Messe-Organisatoren und Kongressveranstalter bestimmen als wichtige Akteure die Rolle, die eine Metropolregion als $h u b$ für relevante Marktin- formationen einnehmen kann. Frankfurt/Rhein-Main steht in dieser Beziehung in einem harten Wettbewerb vor allem mit anderen deutschen Messeplätzen. Nach Zahl der Aussteller, Zahl der ausländischen Aussteller und Zahl der Besucher ist Frankfurt der führende (internationale) Messeplatz in Deutschland. Doch besetzt die Messe in Frankfurt nur wenige Themenbereiche von internationaler Reichweite wie etwa die Internationale Automobilausstellung, die Buchmesse oder das Internationale Treffen für Chemische Technik und Biotechnologie (Achema). Dagegen verfolgt die Messegesellschaft - wie andere auch - eine Internationalisierungsstrategie, indem sie zunehmend Messen als Produkt in anderen Ländern und Kontinenten organisiert. Damit verknüpft sich die Funktion des Messeplatzes als gateway zum nationalen oder mitteleuropäischen Markt mit der eines gateway zu «globalen» Märkten (vgl. Felsenstein, Schamp \& SHACHAR 2002).

Marktfunktionen, Finanzplatz-Funktionen und auch die Funktionen als Innovationsinsel machen eine spezifische Verkehrsinfrastruktur notwendig, die selbst wiederum die Bedeutung eines eigenständigen hubs in europäischen oder globalen Strömen erreichen kann. Wo es um den Austausch von Informationen und Wissen geht, wie in all diesen Funktionsbereichen, ist die Zugänglichkeit im Bereich der Telekommunikation und des Luftverkehrs von eminent großer Bedeutung für die Metropolregion als Knotenpunkt. Infolge der Deregulierung auf den Telekommunikationsmärkten bewirkten die Standortentscheidungen ausländischer Telekommunikationsunternehmen einen Anfangsvorteil für Frankfurt, der heute die Metropolregion zum zweitwichtigsten Telekommunikationsknoten Europas (nach London) macht. Hier befindet sich der zentrale Internet-Knoten für Deutschland, sind mit mehr als 13 Stadtnetzen die meisten lokalen Netzanbieter vorhanden, haben 11 der 13 in Deutschland tätigen globalen Internet-Provider ihr Deutschland-Zentrum und werden $85 \%$ des Internet-Verkehrs in Deutschland abgewickelt (LZB 2001).

Demgegenüber scheint Frankfurts Rolle als Luftverkehrskreuz und hub der Lufthansa als schon traditionell gegeben. Aber in den 1990er Jahren entwikkelte sich auch im deregulierten Flugverkehr ein neuer Wettbewerbsdruck, der einerseits zugunsten des Flughafens Frankfurt wirkt, andererseits zunehmend neue Strategien und lokale Anstrengungen erforderlich zu machen scheint. Frankfurt hat heute in Europa den drittgrößten Flughafen nach Zahl der Passagiere (allerdings haben London und Paris mehrere Flughäfen). Mit $50 \%$ aller Passagiere ist es ein wichtiger UmsteigeFlughafen, mit $35 \%$ aller Passagiere aus anderen Kontinenten zudem von interkontinentaler Bedeutung. Während in 25 Jahren (1975-2000) alle deutschen Flughäfen im Vergleich zu Frankfurt an Bedeutung verlieren, gewinnt nur München mit dem neuen Flug- 
hafen an nationaler Bedeutung (HAAs 1994). Hubs im Flugverkehr binden einerseits als «unteilbare» Infrastruktur viel Kapital und profitieren andererseits von Skaleneffekten. Der Wettbewerb von Flughäfen wird daher weitgehend von Erweiterungs-Investitionen getragen: Die Errichtung eines zweiten Terminals und die Planung eines dritten, die Errichtung der neuen Cargo-City Süd, die geplante neue Landebahn, aber auch die vor einem Jahr erfolgte direkte Anschließung an das ICE-Netz der Bundesbahn bezeichnen die Anstrengungen, die die gegenwärtigen Noch-Eigentümer Land Hessen und Bund - gegen den teils erheblichen Widerstand von Bewohnern - unternehmen, um die Rolle des Flughafens als europäische Drehscheibe zu bewahren. Angesichts knapper Budgets bedienen sie sich durch die gegenwärtige Teilprivatisierung der Fraport AG eines neuen Finanzierungsweges, an dessen Ende möglicherweise ein internationaler Immobilien- und Betreiber-Konzern von Flughäfen steht. Kooperationen mit Wettbewerbern in Europa (wie mit Schiphol/Amsterdam im Jahr 1999), internationale Beteiligungen an Flughäfen (wie z.B.Athen) bzw. an Betriebsgesellschaften (wie z.B. Lissabon, Nairobi) verweisen auf einen ähnlichen Trend zu dem der Messegesellschaften: Aus Infrastrukturgateways für den nationalen oder mitteleuropäischen Markt werden global players (Flughafen Frankfurt/ Main AG 2000).

\section{Welche Rolle spielt die Metropolregion in Europa?}

Drei Folgerungen lassen sich ziehen:

- Erstens ist die Metropolregion Frankfurt/RheinMain erst jüngst zu einer Knotenregion von europäischer Bedeutung aufgestiegen. Während sich die Marktplatz-Funktion noch weitgehend auf den Raum Deutschland und einige Nachbarländer beschränkt, und auch in Deutschland durchaus konkurrierende Metropolregionen bestehen, hat sich die Region Frankfurt/Rhein-Main in den Funktionsbereichen Finanz- und Luftverkehrsdienstleistungen zunächst zu einem Ort von nationaler Bedeutung entwickelt. Darauf aufbauend wurde die Entwicklung zu einem $h u b$ von europäischer Bedeutung im vergangenen Jahrzehnt möglich, nachdem beide Märkte in Europa weitgehend dereguliert wurden.

- Damit hat sich zweitens eine Spezialisierung auf drei Knotenfunktionen durchgesetzt, die der Region einen Vorsprung vor anderen zumindest in Deutschland sichert. Der mehrfache Hinweis auf München - als Technologie-Stadt und neues Luftverkehrskreuz - macht deutlich, dass der Spezialisierungsprozess auch andere Metropolregionen von europäischer Bedeutung in Deutschland hervorbringt.

- Drittens zeichnen sich neue «governance»-Strukturen im Verhältnis zu anderen europäischen Metro- polregionen $\mathrm{ab}$, die dem Verhältnis konkurrierender multinationaler Unternehmen gleichen. Die mächtigen Akteure in der Metropolregion sehen sich zwar im Wettbewerb mit anderen, streben aber zugleich Kooperation in einigen Funktionsbereichen an. Ko-opetition wird damit zur neuen Form der Bestimmung des Verhältnisses zu anderen Metropolregionen, sei es zwischen Messegesellschaften, Flughafen-Betreibern oder Stadtverwaltungen.

Die in diesem Beitrag angesprochenen Knotenfunktionen der Metropolregion Frankfurt/Rhein-Main sind weiterhin «ungesichert» durch die Konkurrenz auf einem weiteren, europäischen Marktgebiet. Das macht den Aufstieg von Frankfurt/Rhein-Main im Prinzip umkehrbar. Dennoch:jüngst haben Duranton \& Puga (2000) auf die bemerkenswerte Stabilität von Stadtsystemen hingewiesen; nur ausnahmsweise scheinen wenige Städte ihren Rang in einem gegebenen Stadtsystem zu verändern. So sollte es nicht verwundern, wenn die Region Frankfurt/Rhein-Main zu einem Steuerungsknoten von hohem europäischen Rang in einigen Aktivitätsfeldern geworden ist, insgesamt aber ihre Stellung im System deutscher bzw. europäischer Stadtregionen wenig verändert hat. Dies würde eher auf eine sich im europäischen Stadtsystem herauskristallisierende Spezialisierung von Metropolregionen hindeuten als auf eine grundlegende Neuordnung der Hierarchie in Europa.

\section{Literatur}

Bertram, H. (1994): Das Speditions- und Transportgewerbe im Wandel. Probleme einer Branche im Verdichtungsraum Frankfurt. - Geographische Rundschau 46: 298-303.

Castells, M. (1996): The Rise of the Network Society. - = The Information Age: Economy, Society and Culture, vol. 1, Oxford.

Dicken, P. (1998): Global Shift. Transforming the World Economy. - Third Edition, London: Paul Chapman.

DohSE, D. (2000): Technology policy and the regions the case of the BioRegio contest. - Research Policy 29: 1111-1133.

Duranton, G. \& D. Puga (2000): Diversity and Specialisation in Cities: Why, Where and When Does it Matter? - In: Urban Studies 37: 533-555.

EsSER, J. \& E.W. Schamp (2001): Metropolitane Region in der Vernetzung. - Frankfurt am Main (im Druck).

EU, EuropäIsche Kommission (1994): Wettbewerbsfähigkeit und Kohäsion:Tendenzen in den Regionen.Brüssel.

Feldman, M.P. \& D.B. AudretsCH (1999): Innovation in cities: Science-based diversity, specialization and localized competition. - In: European Economic Review 43: 409-429.

Felsenstein, D., Schamp, E.W. \& A. Shachar (2002): 
Emerging nodes in the global system: Frankfurt and Tel Aviv metropolitan areas compared. - Dordrecht (im Druck).

Flughafen Frankfurt am MaIn AG (2000): Geschäftsbericht 1999. - Frankfurt am Main.

Freund, B. (1995): Die Suburbanisierung von Betrieben im Rhein-Main-Gebiet. - In: MeYer, G. (Hrsg.): Das Rhein-Main-Gebiet. Aktuelle Strukturen und Entwicklungsprobleme. - = Mainzer Kontaktstudium Geographie 1, Mainz: 45-54.

FRIEDMANN, J. (1995): Where we stand: a decade of world city research. - In: KNOX, P.L. \& P.J. TAYLOR (eds): World cities in a world-system. - Cambridge: 21-47.

FriedmanN, J. \& U.A. Lehrer (1997): Urban Policy Responses to Foreign In-Migration. The Case of Frankfurt-am-Main. - In: Journal of the American Planning Association 63: 61-78.

Greif, S. \& D. Schmiedl (1998): Patentatlas Deutschland. Die räumliche Struktur der Erfindungstätigkeit. - München: Dt. Patentamt, Referat Statistik.

Grote, M. (2002): Frankfurt - An Emerging International Financial Centre. - In: Felsenstein, D., Schamp, E.W. \& A. Shachar: Emerging nodes in the global system: Frankfurt and Tel Aviv metropolitan areas compared. - Dordrecht (im Druck).

HAAS, H.D. (1994): Europäischer Luftverkehr und der neue Flughafen Münchens. - Geographische Rundschau 46: 274-281.

Keil, R. \& K. Ronneberger (1994): Going up the country: internationalization and urbanization on Frankfurt's northern fringe. - In: Environment and Planning D: Society and Space 12:137-166.

Keil, R. \& K. Ronneberger (2000): The Globalization of Frankfurt am Main: Core, Periphery and Social Conflict. - In: Marcuse, P. \& R. van Kempen (eds): Globalizing Cities. A New Spatial Order? - Oxford: 228-248.

LÄPPLE, D. (2000): persönliche Kommunikation.

Lo, V. (2001): Wissensbasierte Vernetzung im Finanzsektor. - In: EsSer, J. \& E.W. SChamp (Hrsg.): Metropolitane Region in der Vernetzung. - Frankfurt am Main (im Druck).

Lo, V. \& E. W. Schamp (2001): Finanzplätze auf globalen Märkten - das Beispiel Frankfurt am Main. - Geographische Rundschau 53, 7/8:26-31.

Lompe, K., Müller, Th., Rehfeld, D. et al. (1991): Regionale Bedeutung und Perspektiven der Automobilindustrie. Die Beispiele Südostniedersachsen und Südhessen. - Düsseldorf: Hans-Böckler-Stiftung.

LZB (Landeszentralbank in Hessen) (2001): New Economy in Rhein-Main. - = Frankfurter FinanzmarktBericht 39, Frankfurt am Main.

Naschold, F., Soskice, D., Hanck, B. \& U. JÜRgens (1998): Ökonomische Leistungsfähigkeit und institutionelle Innovation - Das deutsche Produktions- und Politikregime im globalen Wettbewerb. - = WZB Jahrbuch 1997, Berlin.
Porter, M. (1991): Nationale Wettbewerbsvorteile: erfolgreich konkurrieren auf dem Weltmarkt. - München: Droemer Knaur.

Rentmeister, B. (2001): Vernetzung wissensintensiver Dienstleister in der Produktentwicklung der Automobilindustrie. - In: EsSER, J. \& E.W. SCHAMP (Hrsg.): Metropolitane Region in der Vernetzung. - Frankfurt am Main (im Druck).

RonNeberger, K. (1995): Von High-Tech-Regionen lernen? - In: Jahrbuch sozialwissenschaftliche Technikberichterstattung 1995. - Göttingen: 19-78.

Ronneberger, K. \& R. KeIl (1995): Außer Atem Frankfurt nach der Postmoderne. - In: Hitz, H. et al.: Capitales fatales: Finanzmetropolen im Umbruch. Zürich und Frankfurt auf dem Weg zum Postfordismus. - Zürich: 286-353.

SASSEN, S. (1999): Global Financial Centers. - In: Foreign Affairs 78: 75-87.

Schamp, E. W. (1999): The system of German financial centres at the crossroads: from national to European scale. - In: Wever, E. (ed.): Cities in Perspective I. Economy, planning and the environment. - Assen/NL: 83-98.

Schamp, E.W. (2001): Globalization and the reorganization of a metropolitan knowledge system: The case of R\&D in Frankfurt/Rhein-Main, Germany. - In: Hayter, R. \& R. Le Heron (eds): Knowledge, Territory and Industrial Space. - Aldershot (im Druck).

SPECHT, J. (1998): The role of Frankfurt/Rhine-Main in the R\&D internationalisation strategies of foreign companies. - Unveröffentlichtes Manuskript, Institut für Wirtschafts- und Sozialgeographie, Goethe-Universität Frankfurt am Main.

SPECHT, J. (1999): Industrielle Forschung und Entwicklung: Standortstrategien und Standortvernetzungen. Münster: Lit.

Sternberg, R. \& Ch. Tamásy (1999): München as Germany's No.1 High Technology Region: Empirical Evidence, Theoretical Explanations and the Role of Small Firm/Large Firm Relationships. - In: Regional Studies 33: 367-377.

UVF (Umlandverband Frankfurt) (1993): Technologieprofil der Region Rhein-Main. - Frankfurt am Main. UVF (Umlandverband Frankfurt) (2000): Region Frankfurt RheinMain.-Statistik-Trends, Stand 10/2000. - Frankfurt am Main.

WisSENSCHAFTSSTATISTIK (Hrsg.) (2000): Forschung und Entwicklung in der Wirtschaft 1997-1999. - Essen.

\section{Zusammenfassung: Der Aufstieg von Frankfurt/ Rhein-Main zur europäischen Metropolregion}

Metropolregionen werden in der aktuellen Globalisierungsdebatte als Entscheidungs-, Kontroll- und Koordinationszentren von internationaler Bedeutung verstanden. Sie «bündeln» entsprechende Knoten (hubs), deren Verortung, funktionale Bedeutung und regionale 
Reichweite die Rolle und den Entwicklungspfad der Metropolregion bestimmen. Frankfurt/Rhein-Main ist erst in den letzten beiden Jahrzehnten in diese Rolle hineingewachsen. Knotenfunktionen bestehen heute in drei Themenfeldern: dem Innovationszentrum, dem Finanzplatz und dem Markt(informations)platz. Im Entwicklungspfad der Metropolregion zeigt sich, daß sie zunächst in nationale Bedeutung hineinwachsen mußten, bevor sie internationale Bedeutung gewinnen konnten. Die Knotenfunktionen der Metropolregion Frankfurt/Rhein-Main in den drei genannten Feldern sind jedoch «ungesichert». Daher muß offen bleiben, ob der Aufstieg von Frankfurt/Rhein-Main zur europäischen Metropolregion Auswirkungen auf das weitere System von Metropolregionen in Europa hat.

\section{Summary: The rise of Frankfurt/Rhine-Main to a European Metropolitan Region}

In the current debate on globalisation, metropolitan regions are considered internationally significant centres of decision-making, control and coordination. These regions «bundle» different nodes (hubs) whose location, size and geographical range determine both the role and the trajectory of the respective metropolitan region. It is only in the last two decades that Frankfurt/Rhine-Main has been seen to take on such a role. Today, the region hosts nodes in three areas: innovation, finance and market information/access. The trajectory of the region shows that the hubs first had to gain national importance before reaching international significance. However, the level of significance of these three nodes is still subject to debate. Therefore, it remains to be seen whether the emergence of Frankfurt/Rhine-Main as a European metropolitan region will have an impact on the wider system of metropolitan regions in Europe.

\section{Résumé: L'évolution de Francfort/Rhin-Main vers une région métropolitaine européenne}

Les régions métropolitaines sont considérées dans le cadre de l'actuel débat sur la mondialisation comme des centres de décision, de contrôle et de coordination de portée internationale. Elles structurent des carrefours - des «hubs» - dont la localisation, la signification fonctionnelle et l'influence régionale déterminent le cheminement de la région métropolitaine. L'espace Francort/Rhin-Main n'est parvenu à jouer ce rôle qu'au cours des deux dernières décennies. Il assume à présent une triple fonction de carrefour: en tant que centre d'innovation, de place financière et de place de marché (d'information). Le façonnement de la région métropolitaine révèle que ces trois fonctions ont dû s'affirmer en premier lieu à l'échelle nationale, avant de connaître un rayonnement international. Ces fonctions de carrefour de la région métropolitaine concernée sont cependant contestables. C'est pourquoi il importe de savoir si l'accession de Franfort/RhinMain au rôle de région métropolitaine européenne a entraîné des répercussions sur le système général des régions métropolitaines en Europe.

Prof. Dr. Eike W. Schamp, Institut für Wirtschafts- und Sozialgeographie, Universität Frankfurt, Dantestrasse 9, D-60054 Frankfurt.

e-mail: schamp@em.uni-frankfurt.de

Manuskripteingang/received/rentrée du manuscrit: 10.7.2001

Annahme zum Druck/accepted for publication/accepté pour l'impression: 31.10 .2001 